\title{
Empresas de capital internacional e o sistema de saúde brasileiro: um estudo sobre terapia renal substitutiva
}

\author{
International capital companies and the Brazilian health system: a \\ study on renal replacement therapy
}

Antonio Pescuma Junior (https://orcid.org/0000-0002-9141-3497) ${ }^{1}$

Daniel Figueiredo de Almeida Alves (https://orcid.org/0000-0003-0080-1919) ${ }^{2}$

Aquilas Mendes (https://orcid.org/0000-0002-5632-4333) ${ }^{3}$

Aylene Bousquat (https://orcid.org/0000-0003-2701-1570) ${ }^{3}$
${ }^{1}$ Programa de PósGraduação em Saúde Pública, Universidade de São Paulo (USP). Av. Dr. Arnaldo 715, Pinheiros. 05406-150 São Paulo SP Brasil. antoniopescuma@ alumni.usp.br

${ }^{2}$ Programa de Residência Médica em Infectologia, Instituto de Infectologia Emilio Ribas. São Paulo SP Brasil.

${ }^{3}$ Departamento de Política, Gestão e Saúde, Faculdade de Saúde Pública, USP. São Paulo SP Brasil.

\begin{abstract}
Since the liberalization of foreign capital in health a new trend has arisen in the process of capital accumulation in the industrial economic complex of health, especially at the levels of outsourced care and Services of Care, Treatment and Diagnosis (SCTD). In order to contribute to the understanding of this dynamic, this article sought to analyze the acquisition process of Renal Replacement Therapy (RRT) clinics by international capital companies in Brazil. To achieve this, a two-stage methodological path was developed; the first consisted in the quantitative-descriptive analysis of the economic type of all the international capital companies that operate in the production of input and technologies and in $R R T$ care services; the second was developed by a qualitative analysis of semi-structured interviews of key actors from the private and public sectors and organized civil society. Therefore, the process of the advance of international capital brings with it a qualitative change in the organization, assistance and financing of RRT. It can be seen that, especially in the cases of complementary public and private services, there is a tendency to restrict care and create an obstacle to the universal right to health.
\end{abstract}

Key words Economics of health, Public health, Health systems, Renal dialysis
Resumo A partir da liberalização do capital estrangeiro na Saúde, engendrou-se uma nova tendência no processo de acumulação de capital no complexo econômico industrial da saúde, sobretudo nos níveis de atenção terciários e serviços de Atendimento, Tratamento e Diagnóstico (SADT). Com o intuito de compreender essa dinâmica, o presente artigo procurou analisar o processo de aquisições de clínicas de Terapia Renal Substitutiva (TRS) pelas empresas de capital internacional no Brasil. Para tanto, foi desenvolvido um percurso metodológico em duas etapas: a primeira consistiu na análise quantitativa-descritiva, de modalidade econômica, da totalidade das empresas de capital internacional que atuam na produção de insumos e tecnologias e nos serviços de assistência em TRS; a segunda foi desenvolvida em uma análise qualitativa de entrevistas semi-estruturadas de atoreschaves provenientes dos setores privado e público e sociedade civil organizada. Portanto, o processo de avanço do capital internacional traz consigo uma alteração qualitativa da organização, assistência e financiamento da TRS. Podemos encontrar, especialmente nos casos dos serviços públicos e privados complementares, uma tendência de restrição à atenção integral e um entrave ao direito universal à saúde.

Palavras-chave Economia da saúde, Saúde pública, Sistemas de saúde, Diálise renal 


\section{Introdução}

No atual momento do capitalismo contemporâneo, marcado pelo processo de acúmulo de capital nas suas diversas formas, tanto no segmento produtivo, como no especulativo, é fundamental a ampliação de sua realização na esfera da circulação para manutenção das taxas de lucro ${ }^{1}$, principalmente do grande capital internacionalizado. As empresas de capital internacional, entendidas como transnacionais, perpetuam a valorização do capital pela diversificação das suas atividades, atuando no âmbito da produção de insumos e tecnologias e na oferta de serviços - ou seja, nas esferas produtiva e de circulação.

Dito de outra forma é de fundamental importância para estas companhias a atuação mundializada nos diversos mercados, com elevada concentração financeira ${ }^{2}$. Estas empresas se organizam no formato de oligopólio, tendo como preocupação fundamental assegurar o retorno financeiro aos seus acionistas, com produção em escala para proporcionar um cenário de expansão. O crescimento mundial dos grupos oligopolistas decorre da concentração financeira internacional, sendo expressa pelo processo de aquisições e fusões destas empresas no mundo ${ }^{2}$.

Nas especificidades brasileiras, o setor saúde é chave nesta tendência contemporânea devido à presença de empresas internacionais, formatadas em modelo de oligopólio, responsáveis pela produção de insumos e tecnologias ${ }^{3}$. Importante ressaltar que é na atenção terciária, uma área de maior intensidade tecnológica (mais especificamente maior composição orgânica de capital) e com maior volume de financiamento público, que as empresas transnacionais focam suas estratégias de atuação ${ }^{4}$. Apesar dessa concentração no nível de atenção terciário, a atenção primária tampouco foge a esta lógica ${ }^{5}$.

Dessa maneira, com a abertura da saúde ao capital estrangeiro, sancionada pela Lei $13.097 / 2015^{6}$, é possibilitado ao capital internacional a aquisição de serviços de saúde privados que estão em situação de complementariedade ao Sistema Único de Saúde (SUS). Dentre os principais avanços do capital estrangeiro podemos destacar a "United Health", que em 2016 adquiriu o Hospital Samaritano, em São Paulo, o Hospital da Bahia, em Salvador, o Santa Joana e o Memorial São José, em Recife e o Hospital Santo Helena, em Brasília. Com este avanço do capital, outras companhias estrangerias como o "Grupo Carlyle" (acionista da Qualicorp) e "Rede DASA" (Diagnósticos América/SA) empregaram análises de viabilidade econômica para incorporar serviços de saúde (hospitais e centros de diagnóstico) ${ }^{7}$.

No caso da medicina diagnóstica, nos Serviços de Atendimento, Tratamento e Diagnóstico (SADT), que movimenta cerca de $\mathrm{R} \$ 32$ bilhões, anualmente, e inclui a diálise ${ }^{8}$, a participação do capital estrangeiro é verificada. As aquisições de clínicas e hospitais pelas empresas estrangeiras expressam a ampliação do domínio do capital internacional sobre os serviços privados de diálise, que estão em situação de complementariedade ao SUS?.

De acordo com os dados do Cadastro Nacional de Estabelecimentos de Saúde (CNES), em 2017, 50\% dos serviços de diálise eram privados. Entretato, o SUS é responsável por $89 \%$ do financiamento da hemodiálise no Brasil ${ }^{10}$. Durante o período de 2002 a 2011, o gasto púlico destinado para a Terapia Renal Substituiva (TRS), em características ambulatoriais, correspondeu a $\mathrm{R} \$$ 9,8 bilhões ${ }^{4}$. Ademais, pelas características clínicas da Doença Renal Crônica (DRC), que impacta $8 \%$ a $16 \%$ da população mundial ${ }^{11}$, no atual horizonte tecnológico a necessidade social por este serviço está assegurada.

Além disso, no Brasil, o segmento da diálise possui 758 serviços com programas para o tratamento de diálise crônica ${ }^{12}$. Isto demonstra uma área com elevado potencial de ampliação de aquisições pelas grandes companhias estrangeiras, que no plano internacional dominam a produção de máquinas de diálise e insumos, com forte concentração financeira e organizadas em oligopólio, principalmente em decorrência de direitos de propriedade e aumento dos preços ${ }^{13}$.

As empresas estrangeiras, fornacedoras de insumos e máquinas, com relação ao seu faturamento em 2018 apresentaram a seguinte participação no mercado no Brasil: Fresenius $40 \%$, B. Braun $25 \%$, Baxter $20 \%$ e Nipro $15 \%$, correspondendo a um volume total de rentabilidade de $\mathrm{R} \$ 2,269$ bilhões, sendo Fresenius com R \$ 900 milhões, Baxter R\$ 450 milhões, B. Braun R\$ 569 milhões e Nipro R\$ 350 milhões ${ }^{14-17}$. De acordo com o BNDES, Assis Med era uma empresa nacional com capacidade de fornecimento de insumos e máquinas no âmbito do mercado nacional ${ }^{18}$, porém, teve suas atividades encerradas em $2016^{19}$.

Ainda, é importante observar que o financiamento dos serviços de diálise é majoritariamente composto pelo fundo público. Como se sabe, o fundo público atua para a valorização do capital dessas empresas estrangeiras, contribuindo indiretamente para suas taxas de lucros. Isto significa 
que o Estado, de forma mais acentuada no capitalismo neoliberal, acaba por atuar no econômico não mais pela gestão direta de serviço ${ }^{20}$, como é o caso do setor saúde, mas por conceder, sistematicamente, incentivos ao setor privado (com grande componente de capital internacional) e como garantidor, por meio do fundo público, das taxas de lucro do setor saúde ${ }^{21}$.

Diante dessa relevância da problemática apresentada para o setor saúde, especificamente para o SUS e para a garantia do direito universal à saúde, o objetivo deste artigo é analisar o processo de aquisições de serviços de Terapia Renal Substitutiva (TRS) pelas companhias internacionais (ou capital internacional) e mapear a configuração destas no Brasil.

Com este intuito, foi realizada uma prospecção de dados econômicos sobre as empresas de capital internacional e uma série de entrevistas semiestruturadas com agentes públicos e privados do setor TRS. Compreendemos que a interpretação conjunta desses dados, de natureza distinta, pode avançar na caracterização mais precisa do processo de aquisição de serviços e da dinâmica de acumulação de capital no setor. Para tanto, foi utilizado como marco teórico-conceitual o marxismo, sobretudo o arcabouço categorial da crítica à economia política e as formulações mais relevantes sobre o capital financeiro (ou fictício) ${ }^{2}$.

\section{Metodologia}

Com o objetivo do estudo, o percurso metodológico foi desenvolvido em duas etapas. A primeira consistiu em uma análise quantitativa-descritiva, de característica econômica, da totalidade das empresas de capital internacional que atuam na produção de insumos e tecnologias e na prestação de serviços assistenciais de Terapia Renal Substitutiva (TRS) no Brasil, que estão em situação de complementariedade ao SUS. Toma-se como base a análise documental de relatórios de faturamento, estrutura da empresa e finanças em geral disponibilizadas em plataformas alimentadas pelas próprias companhias.

Esta totalidade foi apreendida, mais especificamente mapeada, pelo índice "Ebtida" (Earnings before interest, taxes, depreciation and amortization), um indicador de concentração para a avaliação de empresas. O "Ebitda", também conhecido como "Ljida"14-17,22, revela o lucro anterior à cobrança dos juros, impostos, depreciação e amortização, ou seja, além de mapear as empre- sas de capital internacional elucida, parcialmente, a lucratividade e o processo de oligopolização.

$\mathrm{Na}$ segunda etapa, foi realizada uma análise de cunho qualitativa, por meio de entrevistas semi-estruturadas de atores-chaves provenientes do setor privado, setor público e sociedade civil organizada (executivos de companhias de capital internacional, gestores de saúde do setor privado, gestores públicos de saúde estaduais e municipais, gestores de agências de vigilância, médicos nefrologistas e associações de pacientes com DRC) no estado de São Paulo, que concentra a maioria destes serviços.

No total foram realizadas nove entrevistas, no período compreendido entre janeiro e março de 2018, as características dos entrevistados foram: idade acima de 30 anos, ensino superior completo, tempo de experiência (no setor específico) acima de 20 anos e posições de trabalho responsáveis pela direção e processo de decisão. As entrevistas foram gravadas e posteriormente transcritas, subsequentemente foi aplicada uma análise de discurso ${ }^{23}$ dos atores-chaves e dos dados mais significativos para o objetivo do estudo.

Após o desenvolvimento destas duas etapas, foi realizada uma síntese (ou triangulação) das análises quantitativas e qualitativas ${ }^{24-26}$, com intuito de tecer hipóteses sobre as tendências e impactos do avanço do capital internacional no segmento da TRS. Por sua vez, também são discutidos seus possíveis desdobramentos para o sistema público sob a égide do direito universal à saúde.

Sobre as questões éticas, os procedimentos metodológicos foram submetidos para apreciação e aprovado pelo Comitê de Ética em Pesquisa (CEP) da Faculdade de Saúde Pública da Universidade de São Paulo (FSP/USP). Foram cumpridos todos os requisitos éticos descritos pela Resolução CNS 466/2012. Todos os entrevistados tomaram ciência do Termo de Consentimento Livre e Esclarecido (TCLE), assinaram e concordaram com as condições para a condução das entrevistas.

\section{Resultados}

Por meio do índice "Ebtida"22, foram apreendidas a totalidade das empresas internacionais (algumas destas de capital aberto) que atuam no segmento de Terapia Renal Substitutiva (TRS) no Brasil. São elas: "Fresenius Medical Care", "Baxter International Inc.", "B. Braun”, "DaVita Inc” (com a subdivisão operacional "Davita Kidney Care"), 
"Diaverum" e "Nipro Corporation". A seguir, passamos a descrever as respectivas realidades econômicas e as atuações no segmento estudado.

Acompanhia "Fresenius Medical Care" ${ }^{15} \mathrm{e}$ "Baxter International Inc." ${ }^{16}$, são as principais empresas do setor de diálise com atuação direta ou com representantes comerciais no Brasil. Atuam na produção de máquinas para diálise e na elaboração de medicamentos, desde o final dos anos 1980, com desenvolvimento no país de soluções para hemodiálise e diálise peritoneal.

A "Fresenius Medical Care", fundada em 1912, com sede na Alemanha, é uma empresa transnacional que opera em mais de cem países, sendo que no Brasil atua há mais de 25 anos. A empresa apresentou uma perspectiva financeira e produtiva favorável no ano de 2018, particularmente com relação à TRS, com uma produção de 50 milhões de procedimetnos, 333.331 pacientes assistidos, 3.928 clínicas próprias e um "Ebitda" de US\$ 73.880.34 milhões ${ }^{15}$. Por outro lado, apresentou um valor de US\$ 57,51 e US $\$ 36,74$ em janeiro de 2018 e janeiro de 2019, respectivamente, por lote de ações, representando uma desvalorização de $36,11 \%{ }^{27}$.

A "Baxter International Inc.", atua de forma diversificada na produção de produtos para a diálise, realizando suas atividades em 25 países e comercializando para mais de $120 \mathrm{em}$ todo o mundo. A empresa opera em dois setores: produtos hospitalares e serviços específicos para o segmento da TRS. Em 2015, a empresa investiu US\$ 603 milhões; em 2014, US\$ 610 milhões e, em 2013, o investimento foi de US\$ 582 milhões em projetos de pesquisa. Com relação aos resultados financeiros, em 2015, as suas vendas, no mundo, totalizaram US $\$ 10$ bilhões, com um decréscimo de 7\% com relação a 2014 (em razão de oscilações no câmbio). O total dos investimentos foi de US\$ 911 milhões em 2015, e o pagamento de dividendos de US\$ 910 milhões, no mesmo ano, para seus acionistas ${ }^{16}$. Em 2018 a companhia auferiu o indicador "Ebitda" no valor de US\$ 2.384 bilhões. Apresentou um valor de US\$ 72,03 e US\$ 72,49 em janeiro de 2018 e janeiro de 2019, respectivamente, por lote de ações, representando uma pequena valorização de $0,63 \%{ }^{28}$.

A "B. Braun", atua no desenvolvimento de produtos, insumos e equipamentos para os mais diversos segmentos da Saúde, em especial na TRS. No Brasil, possui uma sede em São Gonçalo-RJ e sete sedes regionais em outros estados da federação. Em 2015, empregava 55.719 funcionários e tinha um volume de vendas de 6,129 bilhões no mundo, ainda, em 2017 completou 50 anos no
Brasil2". Com relação ao "Ebitda", em 2018, a B. Braun auferiu US\$ 10.542.860 milhões ${ }^{14}$. A empresa "B. Braun" não possui ações negociáveis no mercado acionário.

A "DaVita Inc.", com uma divisão operacional, a "DaVita Kidney Care", está presente em 12 países, com 208.000 pacientes assistidos em mais de 2.660 centros de diálise. Em 30 de junho de 2018, a "DaVita Kidney Care" operava com serviços administrativos, no gerenciamento de 2.580 centros de TRS localizados nos Estados Unidos, atendendo a aproximadamente 201.000 pacientes. A empresa também opera 253 centros de TRS localizados em 10 países fora dos Estados Unidos, com uma receita consolidada de US\$ 2.847 milhões e um lucro operacional de US\$ 289 milhões, incluindo encargos no total de US\$ 93 milhões no mundo ${ }^{30}$. Em junho de 2018, a companhia obteve um "Ebitda" de US\$ 2.117 bilhões. Entretanto, apresentou um valor de US\$ 78,04 e US\$ 56,13 em janeiro de 2018 e janeiro de 2019, respectivamente, por lote de ações, representando uma desvalorização de $28 \%{ }^{31}$.

A "Nipro Coporation" no Brasil trabalha com a divisão médica, composta por seis unidades especializadas em Hemodiálise, Diabetes, serviços Hospitalares, Endovascular, Cardiopulmonar e serviços Diagnósticos. Essa companhia, em 2018, apresenta uma margem bruta sobre o seu faturamento no mundo de $32,17 \%$, margem operacional de 5,95\%, margem líquida de 3,1\% e retorno sobre os investimentos realizados de $2,41 \%$, com um "Ebitda" de US\$ 551.8138 milhões ${ }^{17,32}$. Apesar disso, apresentou um valor de US\$1.686,00 e US\$ 1.458,00 em janeiro de 2018 e janeiro de 2019, respectivamente, por lote de ações, representando uma desvalorização de $13,5 \%{ }^{33}$.

A "Diaverum" é uma dos principais empresas de TRS e a maior prestadora de serviços independente da Europa. As operações atualmente abrangem 18 países e atendem a uma base de pacientes assistidos com mais de 33.000 indivíduos. $\mathrm{Na}$ América Latina, essa empresa atua em quatro países, Argentina, Brasil, Chile e Uruguai, com 2.000 empregados e 6.500 pacientes. Na Argentina, em 2018, mais de US\$ 15 milhões foram investidos na clínica "ITAC", que conta com 50 leitos e 4 salas de operação, e oferta 4.500 procedimentos por ano, incluindo transplantes, serviços de radiodiagnóstico e outras cirurgias ${ }^{34}$. A "Diaverum" apresentou uma valorização de seu "Ebitda" de 13\% em 2017 com relação ao ano de 2016, porém, não é uma empresa de capital aberto.

Com relação às clínicas de TRS no Brasil, as empressas internacionais se comportam de ma- 
neira diversa. As companhias "Fresenius Medical Care" e "DaVita Inc" possuem a maior quantidade dentre as de capital internacional, por sua vez a "Nipro Corporation" e a "B. Braun" somente produzem insumos e equipamentos, portanto, não participando diretamente dos serviços de TRS ${ }^{35,36}$. O Quadro 1 sintetiza este cenário.

Sobre os dados qualitativos, neste estudo, foram priorizados os discursos que desenvolvem a temática do avanço do capital internacional, sobretudo nas aquisições de clínicas de TRS. Conforme os critérios de seleção, foram entrevistados: Gestor Público Muncipal (GPM), Gestor Público Estadual (GPE), Gestor Privado (GPr), Gestor da Vigilância Sanitária (GVS), Executivo de Empresa Internacional (EMP), Gestor de Serviço de Diálise Público (SDP), Gestor de Serviço de Diálise Privado (SDPr), Membro de Associação de Paciente com DRC (APR) e Médico da Sociedade de Médicos Nefrologistas (SM).

De acordo com o discurso do Executivo de Empresa Internacional (EMP), é possível apreender que o movimento de aquisições de clínicas de TRS faz parte do escopo destas companhias internacionais e com particular interesse sobre o financiamento advindo do fundo público. Dessa maneira, temos que de acordo com o entrevistado EMP:

[...] é atrativo gerenciar os serviços, com maior facilidade para pleitear um aumento no financiamento público e por ter os requisitos de "qualidade" exigidos pelos convênios para sua operacionalização.

Além disso, já existe a estrutura institucional com este fim: "Temos uma área de aquisições bem estruturada para compra de clínicas de hemodiálise, os preços dos serviços são atrativos e possuímos uma vantagem cambial". Outro aspecto evidenciado, é a atuação pactuada entre as empresas de capital internacional, característica de mercados oligopolizados, conforme a seguinte fala: "[...] operamos com acordos; entre nós, algumas empresas se dedicam à aquisição de clínicas de maior rentabilidade, outras ao varejo, com a compra de elevadas quantidades".

O Gestor Privado (GPr) e Gestor de Serviço de Diálise Privado (SDPr) demonstram percepções subjetivas divergentes acerca deste avanço sobre as clínicas. O entrevistado GPr, com certa preocupação, comenta: "Bem, eu perdi algumas

Quadro 1. Distribuição de Clínicas de TRS (Terapia Renal Substitutiva) gerenciadas por empresas internacionais no Brasil em 2018.

\begin{tabular}{|c|c|c|}
\hline $\begin{array}{c}\text { Empresas } \\
\text { Internacionais }\end{array}$ & Clínicas & Quantidade \\
\hline $\begin{array}{l}\text { Fresenius Medical } \\
\text { Care }\end{array}$ & $\begin{array}{l}\text { Centro de Nefrologia e Diálise Fresenius Jardins (SP), Centro de Nefrologia } \\
\text { e Diálise Fresenius Perdizes (SP), Centro de Nefrologia e Diálise Fresenius } \\
9 \text { de Julho (SP), CDR Centro (RJ), CDR Vicente de Carvalho (RJ), CDR } \\
\text { Cascadura (RJ), CDR Botafogo (RJ), CDR Taquara (RJ), CDR Anil (RJ), } \\
\text { CDR São Lourenco (RJ), CDR João Mereti (RJ), CDR Nova Iguaçu (RJ), } \\
\text { CDR Barra do Piraí (RJ), CDR Barra Mansa (RJ), CDR Macaé (RJ), Pró-Rim } \\
\text { (RJ), Clinefron (RJ), Clinemge (MG), Instituto Mineiro de Nefrologia (MG), } \\
\text { Nefron (MG), Clínica do Rim (PE), Nefroclínica (PE), Nephron Mix Park } \\
\text { (DF), Nephron Taguatinga (DF), Nephron Gama (DF). }\end{array}$ & 25 \\
\hline $\begin{array}{l}\text { Baxter } \\
\text { International Inc. }\end{array}$ & Clínica Núcleo (MG), Clínica Cauchioli (SP), Baxter Hospitalar (SP). & 3 \\
\hline DaVita Inc. & $\begin{array}{l}\text { Araporanga (PR), Araraquara (SP), Botafogo (RJ), Centro de Acesso Vascular } \\
\text { São Paulo (SP), Jardim das Imbuias (SP), Jardim Itapecerica (SP), João Dias } \\
\text { (SP), Londrina (PR), Meireles (CE), Mondubim (CE), Niterói (RJ), Penha } \\
\text { (SP), Perdizes (SP), Recife (PE), Rolândia (PR), Salvador (BA), Santos (SP), } \\
\text { São Gerardo (CE), Uberlândia (MG), Santo André (SP), Guarulhos (SP), } \\
\text { Taubaté (SP), São José dos campos (SP). }\end{array}$ & 38 \\
\hline Diaverum & $\begin{array}{l}\text { Assitência Médica e Nefrológica de Guarulhos (Ameneg) (SP), Clínica de } \\
\text { Nefrologia de Sergipe (Clinese) (SP) }\end{array}$ & 2 \\
\hline $\begin{array}{l}\text { Nipro } \\
\text { Corporation }\end{array}$ & $\begin{array}{l}\text { Sem clínicas no Brasil. Produção de insumos e equipamentos, Fábrica em } \\
\text { Sorocaba (SP) }\end{array}$ & 0 \\
\hline B. Braun & $\begin{array}{l}\text { Sem clínicas no Brasil. Produção de insumos e equipamentos, Sede em São } \\
\text { Gonçalo (RJ) }\end{array}$ & 0 \\
\hline
\end{tabular}

Fonte: Elaborado pelos autores, baseado em dados extraído das homepages: https://www.davita.com/br/find-a-center/; https:// www.nephrocare.pt/clinicas-de-dialise.html. 
clínicas para transnacionais, pois elas gerenciam o recebimento dos convênios e seguradoras". Entretanto, a existência de poucas empresas internacionais não impulsiona o mercado no sentido de uma "concorrência plena", pelo contrário, segundo o entrevistado SDPr: "Agora, o oligopólio não é bom nunca, em nenhum serviço. Acredito que não pode ser só empresa estrangeira" e que pode contribuir para a piora da assistência em TRS “[...] bem, eu vejo com preocupação porque, uma vez que pode ter uma queda na qualidade do atendimento, diminuição do número de profissionais". Porém, a partir da preponderância desse pensamento na gestão dos serviços de saú$\mathrm{de}^{37}, \mathrm{SDPr}$, contraditoriamente, faz uma defesa das aquisições pelo capital internacional:

[...] com as aquisições, haverá uma maior competitividade, com o aumento dos graus de eficiência na gestão, diminuicão dos gastos, um "melhor relacionamento" com os convênios e seguradoras.

De certa maneira, uma dinâmica similar pauta a subjetividade do discurso do Gestor de Serviço de Diálise Público (SDP), Gestor Público Municipal (GPM), Gestor Público Estadual (GPE) e Gestor da Vigilância Sanitária (GVS). Na interpretação do entrevistado (SDP), o que poderia estar associado à redução de custos e na ampliação do acesso, está relacionado à outra necessidade: "[...] quando elas tiverem um grande número de unidades de diálise, terão um poder de pressão maior até para conseguir um preço melhor para as sessões de hemodiálise pagas pelo SUS".

Outro aspecto, ancorado no direito universal a Saúde, pode ser percebido pelo entrevistado (GPE). Com preocupação ele declara: "[...] e se de repente uma dessas transnacionais comprar todos os serviços do município do Brasil e ela não quer atender SUS, eu fico com um problema enorme na mão". Para complementar, afirma: "A entrada das transnacionais é um elemento preocupante, não há uma certeza se os pacientes SUS dependentes terão atendimento no futuro". Por sua vez, o entrevistado GPM, de maneira subjetiva, elucida a atual tendência das aquisições de ativos pelas empresas internacionais: "[...] hoje os serviços de diálise estão com o financiamento defasado, as clínicas estão tendo prejuízo". Apesar desta preocupação, com lastro concreto, e crítica das aquisições por parte dos gestores públicos, existe a naturalização dessa dinâmica (como algo irreversível), como demostra o entrevistado GVS: "[...] acredito que deva ter uma distribuição entre os serviços, para não ter um único tipo de prestador”.

Curiosamente, a sociedade civil organizada, aqui representada pelo Médico da Sociedade de
Médicos Nefrologistas (SM) e Membro da Associação de Pacientes com DRC (APR), é menos crítica em relação ao processo de aquisições de clínicas de TRS pelo capital internacional. Por exemplo, sob o "guarda-chuva" da imparciabilidade das "leis naturais" que regem o mercado, SM declara: "[...] é uma lei de mercado, não é negativa e nem positiva". Enquanto isso, alijando da determinação do processo saúde-doença a relação entre a gestão do serviço e as necessidades dos usuários em TRS, APR afirma: "Não há problema, contanto que os pacientes SUS e de convênios continuem sendo atendidos".

\section{Discussão}

No panorama internacional, o movimento de aquisições de serviços de Terapia Renal Substitutiva (TRS), nos Estados Unidos (EUA), já está consolidado e apresenta uma receita anual de entorno de US $\$ 25$ bilhões. No atual cenário, "Fresenius Medical Care" e "DaVita Inc." dominam 92\% deste mercado, configurando uma tendência monopolista, e a primeira com fatia de $33 \%$ da produção de máquinas e insumos ${ }^{38}$. No Brasil, ocorre uma fase inicial deste processo de concentração de capital, em características oligopolizadas, nas empresas de capital internacional ${ }^{39-43}$.

Portanto, é possível derivar que a aquisição de clínicas é um reflexo do poderio econômico das empresas de capital internacional no segmento da TRS, com elevada concentração financeira e com controle (quase total) no fornecimento de máquinas e insumos para o segmento. Por consequência, a elevada concentração do serviços de diálise, coordenados pelas companhias internacionais oligopolizadas, colabora para o aumento dos preços dos procedimentos e da taxa de lucro $^{44}$. A concentração dos serviços otimiza os custos, com maior rentabilidade ${ }^{45}$.

De certa maneira, essa unidade contraditória entre as necessidades da acumulação de mais valor (aumento da composição orgânica de capital e diminuição relativa da força de trabalho) e as necessidades impostas pelas condições materiais da produção de saúde nos serviços de TRS (um emprego intensivo de força de trabalho altamente qualificada), foi captada de maneira subjetiva nas entrevistas. Curiosamente, nos "Gestor de Serviços Público de Diálise" e, especialmente, nos "Gestor de Serviço Privado de Diálise" (SPDr) encontramos a forma discursiva mais definida deste conceito. Justamente, se podemos hipotetizar, pelas necessidades imediatas de organizar a 
assistência aos usuários em TRS, o que pode permitir uma compreensão subjetiva mais aguçada dessa contradição.

Outro aspecto observado na subjetividade do discurso presentes nas entrevistas é o fundo público como garantidor das taxas de lucro do capital internacional, atuando como uma contra-tendência à queda da taxa de lucro do capital produtivo, como na perspectiva de $\operatorname{Marx}^{46-49}$. Uma vez que este capital internacional controla o fornecimento de máquinas e insumos (esfera produtiva) e avança sobre a operacionalização das clínicas de TRS (esfera de circulação, ou na esfera de realização do valor), o que permite uma capacidade ampliada de controle dos preços e, por consequência, do financiamento público ${ }^{4}$. Dessa maneira, neste ambiente, quem dita às necessidades sociais em TRS tende a serem os oligopólios de capital internacional, sendo isto um dos desdobramentos da liberalização do capital estrangeiro na saúde.

Ademais, as entrevistas colaboraram para o entendimento deste cenário a partir dos depoimentos dos principais atores que participam na gestão da diálise. Ou seja, as empresas internacionais, principais detentoras de conhecimento científico e tecnológico no segmento, iniciaram o processo de aquisições dos serviços de TRS. Além disso, o cenário atual de câmbio flutuante favorece as aquisições, os serviços de diálise expressos em dólares ou euros correntes possuem custos de incorporação irrisórios para estas empresas de capital internacional ${ }^{50}$. Dessa maneira, a valorização do capital fictício, expresso nos mercados de ações, tem os ganhos futuros assegurados com as aquisições, sendo uma condição fundamental para a rentabilidade das ações representativas destas empresas ${ }^{2}$.

Neste sentido, o arcabouço teórico de Chesnais $^{2}$ é relevante para compreender a nova forma que essas empresas assumem no capitalismo contemporâneo. Especialmente em relação às suas atividades de diversificação, de comercialização de seus produtos, de investimentos realizados no âmbito produtivo e dos resultados operacionais expressos em seus balanços, com reflexos no mercado acionário (ou fictício). Ou seja, a preponderância das empresas de capital internacional na oferta de insumos e máquinas para a diálise é uma condição fundamental para a lucratividade e para a manutenção de um cenário favorável a suas operações no mercado da TRS.

O desequilíbrio do balanço de pagamentos, expresso pela deteriorização do poder de compra de insumos e maquinário realizado pelas clínicas de diálise que não foram incorporadas pelas grandes companhias, ocasiona um cenário inflacionário no segmento, com a necessidade crescente de aumentos nos volumes de financiamento. Desta forma, muitos serviços, remunerados por procedimentos de TRS, passam por problemas na sua operacionalização, condição fundamental para novas aquisições de clínicas pelo capital internacional. Esse processo de incorporações proporciona uma concentração produtiva na oferta, delineando-se uma estrutura propícia para as empresas com maior poder econômico, tanto no ambiente produtivo, como no especulativo, sendo um movimento do capital no seu processo de valorização ${ }^{51}$.

Esses fatores, apresentam aos usuários a possibilidade, em um futuro próximo, de restrição ou a instabilidade do acesso aos serviços de TRS. Neste cenário, as empresas estrangeiras, a princípio, terão duas alternativas: aumentar o parque instalado e oferecer o tratamento a todos os pacientes do SUS ou disponibilizar o tratamento para um maior número de pacientes de convênios e seguradoras. Com relação à primeira alternativa, haverá um maior poder de negociação das empresas de capital internacional perante o SUS. Sobre a segunda alternativa, há uma questão duvidosa perante o comportamento das seguradoras e convênios, principalmente, com relação a capacidade de financiamento dos serviços de diálise.

De todo modo, esse processo de avanço do capital internacional traz consigo uma alteração qualitativa da organização, acesso, assistência, custos e financiamento da Terapia Renal Substituiva (TRS) no Brasil. Seja este nos serviços públicos, nos serviços privados em situação de complementariedade ou nos serviços privados suplementares. Podemos encontrar, especialmente nos casos dos serviços públicos e privados em situação de complementariedade, uma tendência de restrição à atenção integral dos usuários em TRS e um entrave ao direito à saúde sob a ègide da universalidade.

\section{Considerações finais}

A partir da análise quantitativa e qualitativa utilizada neste estudo, procurou-se apreender a dinâmica das aquisições de serviços de Terapia Renal Substitutiva (TRS) pelas empresas de capital internacional no Brasil. Este processo não é uma tendência "natural" das leis do mercado, pelo contrário, é uma das expressões materiais 
contemporâneas da acumulação de capital, sendo um dos reflexos da liberalização do capital estrangeiro na Saúde.

Nas especificidades desse fenômeno podemos encontrar o avanço do capital internacional, que já detém o controle da produção de tecnologia e insumos, sobre a esfera da circulação (ou de realização do valor). O impacto deste processo sobre o setor Saúde pode ser observado na apropriação do fundo público, que vem atuando como garantidor da acumulação de capital nesse segmento e, por sua vez, como contratendência à queda da taxa de lucro do capital produtivo.

Em suma, o Estado, ao atender as necessidades do capital internacional, coloca em risco o acesso universal e a atenção integral de usuários em TRS. Ou seja, alija-se da centralidade da atenção as necessidades de Saúde da população.

Apesar da relevância das tendências e hipóteses descritas, este artigo apresenta como limites o caráter introdutório das análises econômicas das empresas de capital internacional. Isto se deve à dificuldade de compilação destes dados, em virtude da pouca disponibilidade dos mesmos.

Por fim, com a necessidade de expandir a análise realizada sobre o segmento de TRS no Brasil, podemos indicar como temática de futuros estudos o mapeamento do acesso dos usuários aos serviços adquiridos pelas empresas de capital internacional e o impacto desta tendência sobre o financiamento da Saúde Pública compreendida enquanto direito universal.

\section{Colaboradores}

A Pescuma Jr. realizou a pesquisa, concepção, metodologia e redação final. DFA Alves contribuiu na metodologia e redação final. A Mendes contribuiu na redação final. A Bousquat contribuiu na concepção, metodologia e redação final. 


\section{Referências}

1. Mendes A. Brazilian public health in the context of a State crisis or a crisis ofcapitalism? Saude Soc 2015; 24(Supl. 1):66-81.

2. Chesnais F. A mundialização do capital. São Paulo: Xamã; 1996.

3. Gadelha CAG. Estudo da competitividade de cadeias integradas no Brasil: impactos das zonas livres de comércio. Nota Técnica Final. Campinas: IE/Neit/Unicamp, MCT/Finep, MDIC; 2002.

4. Pescuma Jr A. O financiamento da média e alta complexidade do SUS: uma análise dos recursos financeiros da terapia renal substitutiva [dissertação]. São Paulo: Pontifícia Universidade Católica de São Paulo; 2013.

5. Mendes A, Carnut L, Guerra LDS. Reflexões acerca do financiamento federal da Atenção Básica no Sistema Único de Saúde. Saude Debate 2018; 42(n. esp. 1):224243.

6. Brasil. Lei no 13.097, de 19 de janeiro de 2015. Reduz a zero as alíquotas da Contribuição para o PIS/PASEP, da COFINS, da Contribuição para o PIS/Pasep-Importação e da Cofins-Importação incidentes sobre a receita de vendas e na importação de partes utilizadas em aerogeradores e dá outras providências. Diário Oficial da União 2015; 20 jan.

7. Cruz E, Monteiro Neto F. Capital Internacional e suas consequências para a Assistência a Saúde. Rio de Janeiro: Cebes; 2016.

8. Associação Brasileira de Medicina Diagnóstica (Abramed). O DNA do Diagnóstico [Internet]. 2018 [acessado 2019 jun 22]. Disponível em: http://online.pubht$\mathrm{ml} 5 . \mathrm{com} / \mathrm{pmiv} / \mathrm{qrgv} /$.

9. Andrade A, Rossetti JP. Governança Corporativa: fundamentos, desenvolvimento e tendências. São Paulo: Atlas; 2009.

10. Szuster DAC, Caiaffa WT, Andrade EIG, Acurcio FA Cherchiglia ML. Sobrevida de pacientes em diálise no SUS no Brasil. Cad Saude Publica 2012; 28(3):415424.

11. Jha V, Garcia-Garcia G, Iseki K, Li Z, Naicker S, Plattner B, Saran R, Wang AY-M, Yang C-W. Chronic kidney disease: global dimension and perspectives. Lancet 2013; 382 (9888):260-272.

12. Thomé FS, Sesso RC, Lopes AA, Lugon JR, Martins CT. Inquérito Brasileiro de Diálise Crônica 2017. J Br Nefrol 2019; 41(2):208-214.

13. Aragão E, Abreu G, Loureiro S. Inovações tecnológicas em saúde e suas implicações para a equidade: evidências da desigualdade na distribuição de equipamentos de diagnóstico no Brasil. In: Costa LS, Bahia L, Gadelha CAG. Saúde, desenvolvimento e inovação. Rio de Janeiro: Cepesc; 2015.

14. B Braun. Annual Report 2018 - ole, a life story. Germany: Hessen; 2018.

15. Fresenius Medical Care. Overview [Internet]. [acessado 2019 set 30]. Disponível em: http://www.freseniusmedicalcare.com/en/investors/operating-figures/.

16. Baxter International Inc. Overview [Internet]. [acessado 2019 set 30]. Disponível em: http://investor.baxter. com/phoenix.zhtml? $\mathrm{c}=86121 \& \mathrm{p}=$ irol-reportsannual.

17. Nipro Corporation. Annual report 2018 [Internet]. [acessado 2020 jun 16]. Disponível em: https://www. nipro.co.jp/en/ir/library/document/65th/annual_report.pdf.
18. Banco Nacional de Desenvolvimento Econômico e Social (BNDES). Equipamentos para Hemodiálise [Internet]. [acessado 2020 jun 16]. Disponível em: https:// web.bndes.gov.br/bib/jspui/bitstream/1408/3068/2/ BS\%2012\%20Equipamentos\%20para\%20Hemodi\%C3\%Allise_P.pdf.

19. Agência Nacional de Vigilância Sanitária (Anvisa). Lista de operadoras que tiveram seu registro cancelado em fevereiro de 2016 [Internet]. [acessado 2020 jun 16]. Disponível em: http://www.ans.gov.br/images/ stories/Plano_de_saude_e_Operadoras/informacoes_avaliacoes/canceladas_24-02-2016.pdf.

20. Dardot P, Laval C. A nova razão do mundo: ensaio sobre a sociedade neoliberal. São Paulo: Boitempo; 2016.

21. Salvador E. Fundo Público e Seguridade Social no Brasil. São Paulo: Cortez Editora; 2010.

22. Marks HK, Slee RT, Blees CW, Nall MR. Financial Analysis. In: Marks HK, Slee RT, Blees CW, Nall MR. Handbook for investment banking and business consulting. Nova Jersey: Willey; 2012. p. 139-150.

23. Bauer MW, Gaskell G. Pesquisa qualitativa com texto, imagem e som - um manual prático. $8^{\mathrm{a}}$ ed. Petrópolis: Vozes; 2010.

24. Denzin NK. Sociological Methods. Nova York: McGraw-Hill; 1978.

25. Webb EJ, Campbell DT, Schwartz RD, Sechrest L. Unobtrusive Measures: Nonreactive Measures in the Social Sciences. Chicago: Rand McNally; 1966.

26. Patton MQ. Enhancing the quality and credibility of qualitative analysis. Health Serv Res 1999; 34(5):11891208.

27. Investing Brasil. Fresenius Medical Care $A G$ \& $C O$ (FMEG) [Internet]. [acessado 2019 out 30]. Disponível em: https://br.investing.com/equities/freseniusmedi.

28. Investing Brasil. Baxter International Inc. (BAX) [Internet]. [acessado 2019 out 30]. Disponívvel em: https://br.investing.com/equities/baxter-intl.

29. B Braun. Annual report 2015 - ole, a life story. Germany: Hessen; 2015.

30. DaVita Inc. United States Securities and Exchange Commision [Internet]. [acessado 2019 set 30]. Disponível em: http://investors.davita.com/static-files/ b459523d-726b-4fdf-96c3-be6cbb69040f.

31. Investing Brasil. DaVita Heath Care Partners Inc (DVA) [Internet]. [acessado 2019 out 30]. Disponívvel em: https://br.investing.com/equities/davita-inc.

32. Nipro Corporation. Market Screener [Internet]. [acessado 2019 set 30]. Disponível em: https:// www.marketscreener.com/NIPRO-CORPORATION-6491866/financials/.

33. Investing Brasil. Nirpo Corp. [Internet]. [acessado 2019 set 30]. Disponível em: https://br.investing.com/ equities/nipro-corp.

34. Diaverum. Comunicado de Prensa [Internet]. [acessado 2019 set 30]. Disponível em: http://www.diaverum.com/es-ES/Corporate-Menu/Global-News/ Noticias-relacionadas/2018/7/press-release-diaverum -invests-in-state-of-the-art-institute-for-complextransplants-d.institute-itac/.

35. DaVita Inc. Tratamento Renal [Internet]. [acessado 2019 nov 30]. Disponível em: https://www.davita. $\mathrm{com} / \mathrm{br} /$ find-a-center/. 
36. Nephro Care. Clíncias de Diálise [Internet]. [acessado 2019 nov 30]. Disponível em: https://www.nephrocare.pt/clinicas-de-dialise.html.

37. Alves DFA, Carnut L, Mendes A. Dimensionamento da 'economia política' na 'economia da saúde': para refletir sobre o conceito de sustentabilidade. Saude Debate 2019; 43(n. esp. 5):145-160.

38. Baker RS. The U.S. health care system is full of monopolies [Internet]. Axios; 2019 [acessado 2019 nov 30]. Disponível em: https://www.axios.com/health-carecosts-monopolies-competition-hospitals-9839f396c95d-4792-b106-663a727eflf4.html.

39. Pharmexx Brasil. Baxter volta as compras e adquire clínica de hemodiálise no Brasil [Internet]. 2017 [acessado 2019 nov 30]. Disponível em: http://www.pharmexxbrasil.com.br/noticias/baxter-volta-as-compras -e-adquire-clinica-de-hemodialise-no-brasil/.

40. Fórum Saúde Digital. Multinacional alemã vai investir $R \$ 300$ milhões no segmento da diálise [Internet]. 2017 [acessado 2019 nov 30]. Disponível em: http:// forumsaudedigital.com.br/multinacional-alema-vaiinvestir-r-300-milhoes-no-segmento-de-dialise.

41. Folha de São Paulo. Multinacionais buscam consolidação do setor de hemodiálise no Brasil [Internet]. 2019 [acessado 2019 nov 30]. Disponível em: https://www1. folha.uol.com.br/colunas/mercadoaberto/2018/07/ multinacionais-buscam-consolidacao-do-setor-de -hemodialise-no-brasil.shtml.

42. Diário de Suzano. Empresa Sueca compra institutos de nefrologia de Suzano e Mogi [Internet]. 2018 [acessado 2019 nov 30]. Disponível em: https://www.diariodesuzano.com.br/cidades/empresa-sueca-compra-institutos-de-nefrologia-de-suzano-e-mogi/45654/.

43. Associação Brasileira do Centros de Diálise e Transplante. DaVita tratamento renal adquire operação de agudos do grupo transrim de São Paulo [Internet]. 2019 [acessado 2019 nov 30]. Disponível em: https://www. abcdt.org.br/davita-tratamento-renal-adquire-operacao-de-agudos-do-grupo-transrim-de-sao-paulo/.

44. Cutler DL, Dafny LS, Ody CJ. How does competition impact the quality of price of outpatient service facilities?: A case study of the U.S. Dialysis Industry. Northwestern University; 2017.
45. Pozniak AS, Hirth RA, Banaszak-Holl J, Wheeler JRC. Predictors of chain acquisition among independent dialysis facilities. Health Serv Res 2010; 45(2):476-496.

46. Marx K. O capital: crítica da economia política: livro 1: processo de produção do capital. 2a ed. São Paulo: Boitempo; 2017.

47. Behring ER, Boschetti I. Politica social: fundamentose história. 9a ed. São Paulo: Cortez; 2011.

48. Harvey D. O enigma do capital e as crises do capitalismo. São Paulo: Boitempo; 2010.

49. Mendes A, Carnut L. Capitalismo contemporâneo em crise e sua forma política: o subfinanciamento e o gerencialismo na saúde pública brasileira. Saude Soc 2018; 27(4):1105-1119.

50. Cesário BB, Costa LS, Maldonado JMSV, Vargas MA. Vulnerabilidade do SUS em relação à variação cambial: análise da dinâmica de importações de medicamentos e equipamentos de saúde entre 1996 e 2014. Saude Debate 2017; 41(113):441-456.

51. Chesnais F, Sauviat C. The financing of innovation -related investment in the contemporary global finance-dominated accumulation regime. In: Cassiolato JE, Lastres HMM, Maciel ML. Systems of innovation and development: evidence from Brazil. Cheltenham: Edward Elgar; 2003. p. 61-118.

Artigo apresentado em 01/04/2020

Aprovado em 29/06/2020

Versão final apresentada em 02/07/2020

Editores-chefes: Romeu Gomes, Antônio Augusto Moura da Silva 